\title{
USING INDEPENDENT COMPONENTS ANALYSIS TO CALCULATE INTRAVASCULAR CONTRAST AGENT CONCENTRATION IN PROSTATE CANCER
}

\author{
Hatef Mehrabian $^{* 1,2}$, Masoom A. Haider ${ }^{3}$, Anne L. Martel ${ }^{1,2}$ \\ ${ }^{1}$ Department of Medical Biophysics, University of Toronto, Toronto, ON, Canada \\ ${ }^{2}$ Physical Sciences, Sunnybrook Research Institute, Toronto, ON, Canada \\ ${ }^{3}$ Dept. of Medical Imaging, Sunnybrook Health Science Center, University of Toronto, Toronto, Canada
}

\begin{abstract}
Dynamic contrast enhanced MRI combined with Pharmacokinetic (PK) modeling of prostate tissue provides information about its microenvironment and vascular permeability. A fundamental step in PK analysis is measuring the intravascular contrast agent concentration or the arterial input function (AIF).

In this study a method is introduced to calculate the intravascular concentration in the prostate tissue using an adaptive complex independent components analysis (ACICA) method and to correct this curve for the early phases of the passage of the contrast agent through tumor vasculature. The results are applied to DCE-MR images of the prostate of a 70 year old prostate cancer patient and the calculated $K^{\text {trans }}$ map is examined using tumor location defined by multi-parametric MRI. The results show that there is a high $K^{\text {trans }}$ value in the region in the peripheral zone of the prostate that was hypo-intense in both apparent diffusion coefficient map and T2-weighted MRI. Moreover the results are compared with the parameters derived using a large artery and also corrected artery as the intravascular concentration curve.
\end{abstract}

Index Terms - Prostate cancer, Pharmacokinetic modeling, intravascular concentration, independent component analysis (ICA), Adaptive complex ICA.

\section{INTRODUCTION}

Prostate cancer accounts for $14 \%$ of the total new cancer cases worldwide. It is the sixth cause of cancer death leading to $6 \%$ of total cancer deaths in males [1]. Prostate cancer diagnosis process begins with prostate-specific antigen (PSA) testing followed by digital rectal examination. There is no reliable non-invasive method to differentiate benign and malignant tumors and thus most patients are treated with radical treatment methods [2]. Dynamic contrast enhanced MRI (DCE-MRI) is a noninvasive imaging technique that has the potential to provide information about tumor microenvironment and angiogenesis. DCE-MRI combined with pharmacokinetic models which were developed in the early 1990s enable the estimation of blood perfusion and capillary permeability.

Accurate measurement of the pharmacokinetic (PK) model parameters, however, is currently limited to the research environment as it requires DCE-MRI data with high temporal resolution which is not yet clinically practical [3]. A fundamental step in PK modeling is measuring the intravascular contrast agent concentration or arterial input function (AIF) which describes the passage of a bolus of contrast agent through the vasculature and is used as an input to the PK model. Measuring such a signal in the prostate area is extremely difficult and thus it is usually approximated from a major artery adjacent to the tumor [4], a standard AIF [5], population averaged AIF [6], etc.

In a previous study we proposed a method based on an adaptive complex independent components analysis (ACICA) method to calculate the intravascular concentration in the tissue of interest [7-9]. This method provided a good estimate to the intravascular signal both temporally and spatially. However due to the partial volume effect, high contrast concentration and the low temporal resolution of the images it was unable to recover the true amplitude of the concentration curve in the early phases of the bolus passage which affects the PK parameters.

Fan et al. [10] proposed a reference region based method to correct AIF for its underestimation of the early phases. However his method required knowledge of the contrast agent in an artery, which is not usually available in PK analysis. It also required optimizing for the time lag between the arrival of bolus in the region that the correction was performed and the tissue of interest. In this study we correct the intravascular concentration curve calculated with the ACICA using the reference region-based method [10] and apply it to DCE-MR images of the prostate tissue. Our procedure does not require knowledge of the contrast uptake in an artery outside the tumor, and since it is being performed in the prostate tissue, does not require optimization for the time lag of intravascular curve and the tumor tissue. The PK parameters will be studied in 3 different cases: using the a) concentration in an artery b) corrected artery curve and c) corrected ACICA-based curve.

\section{METHODS}

2.1. Adaptive Complex Independent component analysis Independent component analysis (ICA) is a statistical signal processing method that tries to identify the underlying features, $S$, of an observed time series signal, $Z$ such that $Z=A S$, where columns of $S$ are assumed to be independent 
and $A$ is the mixing matrix. ICA algorithms try to find an unmixing matrix $W$ such that $Y=W Z$ where columns of $Y$ and $S$ are different only in scaling and permutation [11].

We developed and validated an adaptive complex ICA (ACICA) technique to calculate the intravascular contrast agent concentration in the tissue of interest [7-9]. The major assumption in this method was the spatial independence of the intravascular and extravascular MR signals. It also assumed that the distribution of the real and imaginary parts of the MRI signal can be approximated with a linear combination of a number of (usually 3 to 5) generalized Gaussian distributions [8-9] which is given as follows:

$$
p_{y}(y)=\frac{\beta}{2 \alpha \Gamma(1 / \beta)} \exp \left(-\frac{|y|^{\beta}}{\alpha^{\beta}}\right)
$$

where $\Gamma($.$) is the Gamma function. The ACICA method$ first calculates the two model parameters $(\alpha, \beta)$ of the intravascular signal through an expectation maximization framework at each iteration of the ICA process. It then uses this probability density function to derive the non-linearity in ICA algorithm. Finally the non-linearity is used in the fixed point update rule of complex ICA given as $[8,9,12]$ :

$$
\begin{aligned}
w_{k+1} & =-E\left\{G^{*}\left(w_{k}^{H} Z\right) G^{\prime}\left(w_{k}^{H} Z\right) Z\right\} \\
& +E\left\{G^{\prime}\left(w_{k}^{H} Z\right) G^{\prime *}\left(w_{k}^{H} Z\right)\right\} w_{k} \\
& +E\left\{Z Z^{T}\right\} E\left\{G^{*}\left(w_{k}^{H} Z\right) G^{\prime \prime}\left(w_{k}^{H} Z\right)\right\} w_{k}^{*}
\end{aligned}
$$

where $G($.$) is the nonlinearity function and is derived as:$ $\mathrm{G}(\mathrm{z})=\left(\frac{\mathrm{z}}{\alpha}\right)^{\beta / 2}, E$ is the expectation operator, $G^{\prime}, G^{\prime \prime}, G^{*}$ are the first and second derivatives and the complex conjugate of $G$ respectively, $w_{k}$ is a column of unmixing matrix $W$ at iteration $k$, and $w_{k}^{H}$ is Hermitian transform of $w_{k}$.

\subsection{Pharmacokinetic modeling}

The two compartmental extended Tofts model [13] which is comprised of the blood plasma and extravascular extracellular space (EES) compartments was used to analyze the DCE-MRI data and calculate the $K^{\text {trans }}, v_{p}$ and $v_{e}$ parameters for every voxel in the prostate. The equations describing the contrast concentration in compartments are:

$$
\begin{gathered}
v_{e} \frac{d C_{e}}{d t}=K^{\text {trans }}\left(C_{p}(t-\omega)-C_{e}(t)\right) \\
C_{t}=v_{e} C_{e}+v_{p} C_{p}
\end{gathered}
$$

where $C_{t}$ is the concentration of the contrast agent in the tumor tissue, $C_{e}$ is the concentration in the EES, $C_{p}$ is the intravascular contrast concentration also called AIF, $K^{\text {trans }}$ is the volume transfer coefficient, $v_{e}$ is the EES per unit volume of tissue, $v_{p}$ is the plasma volume fraction and $\omega$ is the time lag between blood arriving in the tumor tissue and where the intravascular concentration is being measured or approximated. The time lag is zero for the ACICA-based intravascular concentration but is non-zero for the curve approximated from an artery and has to be optimized.

\subsection{Reference region-based AIF correction}

The intravascular concentration curve was corrected by adapting the reference region (RR)-based method introduced by Fan et al. [10] to our problem. An area of the prostate that showed very little perfusion was selected using the complex ICA results. This region was selected using the independent component (IC) image that corresponds to the intravascular signal. Then, contrast concentration over time was obtained in this region $\left(C_{m}\right)$. The slope of $C_{m}$ was close to zero at times greater than $5 \mathrm{~min}$. Thus, it was assumed that after this time, the EES and intravascular concentrations were close to equilibrium. The $v_{e}$ of this region was calculated as follows [10]: $v_{e} \approx \frac{c_{m}(t)}{c_{p}(t)}$ for $t \geq 5 \mathrm{~min}$, where $C_{p}$ is the intravascular concentration calculated by ACICA (without correction). The value of $K^{\text {trans }}$ for the reference region was then calculated by fitting equation 3 to the $C_{m}$ and $C_{p}$ for times greater than the peak of $C_{p}$. Once $K^{\text {trans }}$ and $v_{e}$ were calculated, the corrected intravascular concentration curve was calculated using equation 4 [10]:

$$
C_{p, \text { corrected }}=\frac{1}{K^{\text {trans }}} \frac{d C_{m}(t)}{d t}+\frac{C_{m}(t)}{v_{e}}
$$

\subsection{Clinical Study}

A 70 year old patient with high PSA and biopsy proven prostate cancer (Gleason score 6), underwent multiparametric MR imaging including T2-weighted imaging, diffusion weighted imaging (DWI, b-value 100/400/1000 $\mathrm{s} / \mathrm{mm}^{2}$ ) and DCE-MRI with a 3T MRI Philips unit. The contrast agent, Magnevist (Gd-DTPA) was injected intravenously with a rate of $4 \mathrm{mMol} / \mathrm{Kg}$.

Dynamic contrast-enhanced MR imaging was performed using 3D spoiled gradient echo (SPGR) acquisition with $\mathrm{TR} / \mathrm{TE}=3.9 / 1.8 \mathrm{~ms}$, Flip Angle $=8^{\circ}, \mathrm{Nx} / \mathrm{Ny} / \mathrm{NEX}=128 / 128 / 2$, FOV $=20 \mathrm{~cm}$, Slice Thickness $=3.5 \mathrm{~mm}$, where 75 images with a temporal resolution of $4.85 \mathrm{~s}$ were acquired over 6 minutes. A sample frame of the DCE-MRI of prostate (the slice where the tumor is located) is shown in figure 1 along with its T2-weighted image and apparent diffusion coefficient (ADC) map generated using the diffusion weighted images.

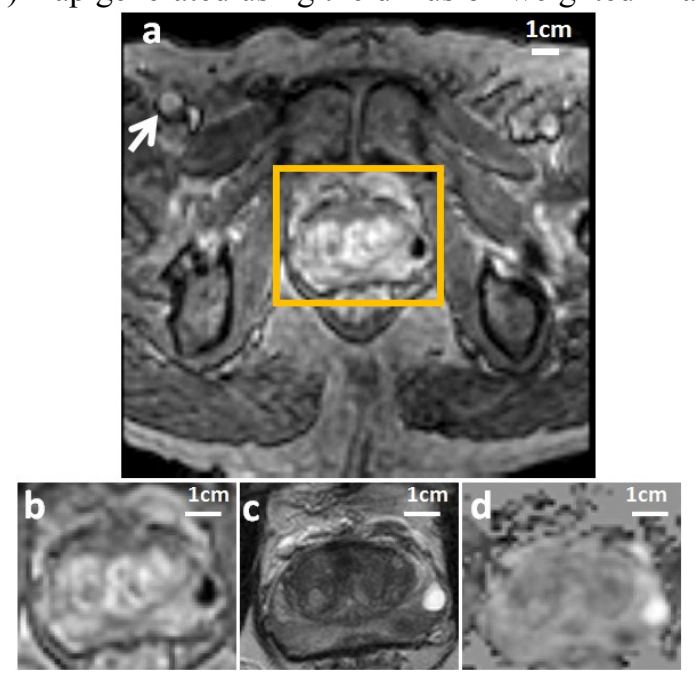

Figure 1. a) Full field of view of the DCE-MR images. This image shows the prostate region (yellow box) and an artery that is used in the analysis (arrow). b) The prostate in the DCE-MR image. c) The T2-weighted image, and d) the ADC map of the prostate. 


\section{RESULTS}

\subsection{AIF measurement}

The complex DCE-MRI data was fed to the ACICA algorithm and the intravascular component was calculated (figure 2). This intravascular signal is then converted to contrast concentration to obtain the AIF for PK analysis.

The intravascular concentration curve that was calculated using ACICA method was then corrected for early phases of the passage of the bolus using the introduced RR-based correction method (shown in figure 2a). The ROI that was used for correction is shown in figure $2 \mathrm{~b}$. As can be seen from the intravascular image (figure 2c) this ROI has little perfusion and thus is suitable for correction. For comparison AIF in a major artery (arrow in figure 1) was also measured and was corrected using the RR-based method (figure 2a).
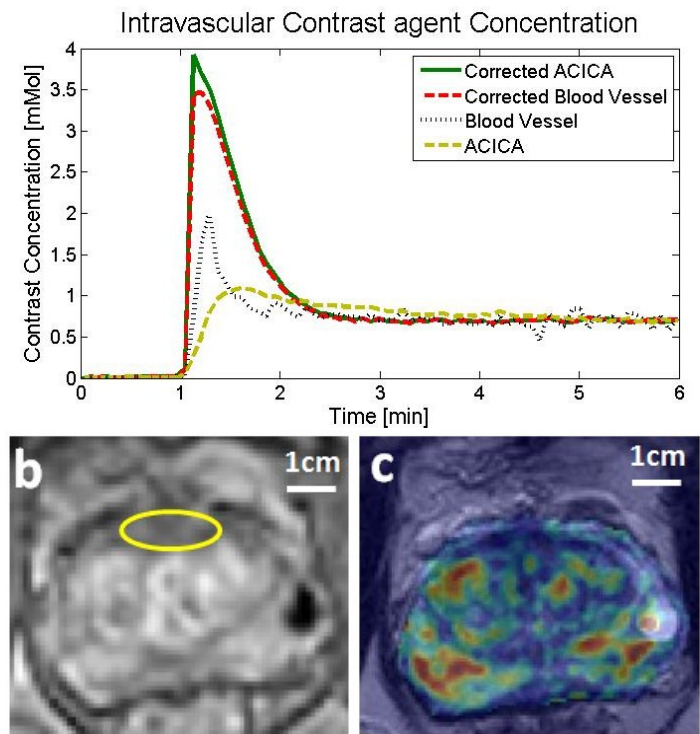

Figure 2. a) The intravascular concentration curve calculated using ACICA before and after correction as well as the curves measured in the artery shown in figure 1 before and after correction. The time lag between the bolus arrival in the artery and prostate is corrected for in the artery curves to enable comparison. b) A sample DCE-MR image where the low perfusion ROI used for correction is shown (ellipse) and c) the intravascular IC image overlaid on the $\mathrm{T} 2$-weighted image of the prostate, corresponding to the intravascular curve of ACICA.

\subsection{Pharmacokinetic Analysis}

The modified Tofts model was used to perform PK analysis where the PK parameters, $K^{\text {trans }}, v_{e}$ and $v_{p}$, were calculated for every voxel. The PK analysis was performed in three cases: using the a) corrected intravascular curve from ACICA, b) AIF from the artery, and c) corrected artery AIF. Figure 3 shows $K^{\text {trans }}, v_{e}$ and $v_{p}$ maps calculated for the first case. These maps are overlaid on the T2-wieghted image. The ADC map and the T2-weighted image of the prostate are also shown in this figure. These images show that there is a hypo-intense area in the peripheral zone (PZ) of the prostate in both T2-weighted image and ADC map.
Such a region is suspicious and could correspond to tumor in the prostate. The $K^{\text {trans }}$ map of the prostate also shows high $K^{\text {trans }}$ values in the same region. Further examinations such as biopsy were required to verify whether this region corresponded to a tumor. Follow up studies confirmed prostate cancer with Gleason score 6. A similar process was performed using the AIF curves measured in the artery both with and without correction and their $K^{\text {trans }}$ maps are shown in figure 4.
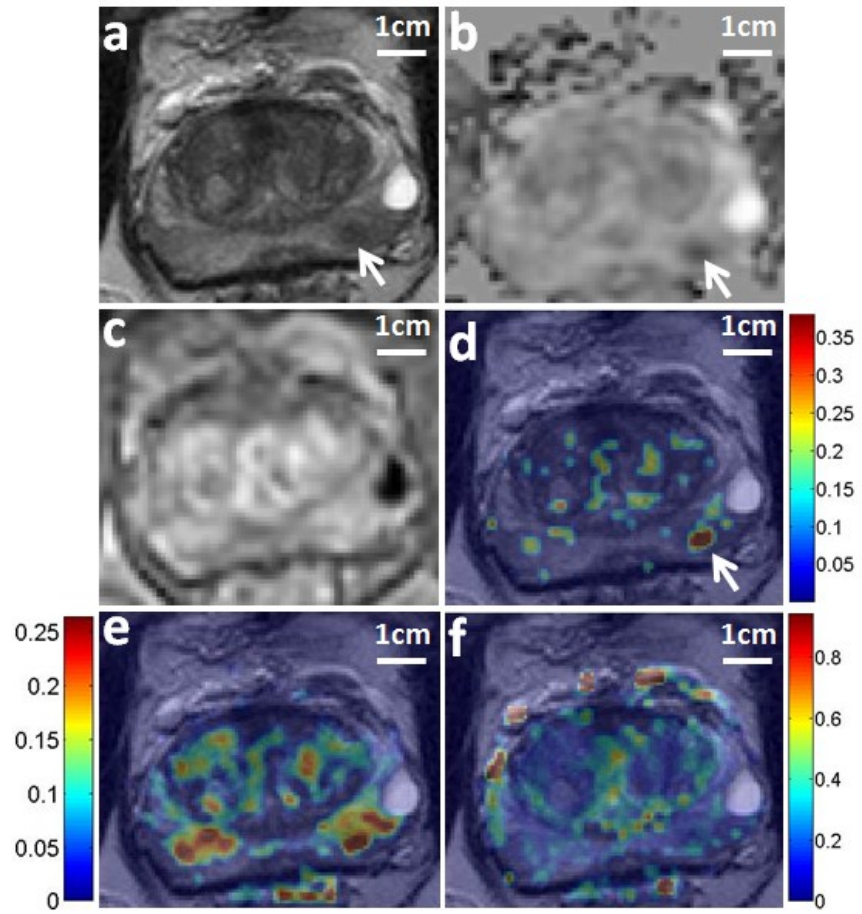

Figure 3. a) The $\mathrm{T} 2$-weighted image of the prostate showing the hypo-intense region in the peripheral zone of the prostate (arrow). b) The ADC map of the prostate showing hypo-intense voxels in the same region as T2-weighted image (arrow). c) A sample DCEMRI image of the prostate. d) The $K^{\text {trans }}$ map calculated for the prostate overlaid on the T2-weighted image. The map was calculated using the corrected intravascular concentration curve derived from ACICA. The map shows high $K^{\text {trans }}$ values in the same anatomical region as the hypo-intense region in $\mathbf{a}$ and $\mathbf{b}$ (arrow). e) The $v_{p}$ and $\mathbf{f}$ ) the $v_{e}$ map overlaid on the T2-weighted image, calculated using the corrected ACICA concentration curve.

\section{DISCUSSIONS}

Pharmacokinetic modeling of tumors provides information about the tumor permeability and blood volume. Such parameters have been shown to be related to prognostic factors. A novel technique was introduced to calculate and correct the intravascular contrast concentration in the prostate area with no requirement for prior information about the PK parameters of the normal tissue or information about contrast uptake in a blood vessel.

As can be seen in figure 2, the concentration curve calculated by ACICA underestimated the uptake in the early phases of the bolus passage through the prostate vasculature. This could be due to high partial volume effect and also 
saturation of the signal that is being measured. Moreover the contrast uptake in an artery is unable to measure the contrast concentration in the early phases (it underestimates the peak) and fails to capture the profile of the intravascular curve (its shape is different from that of the prostate). Thus correction is necessary to calculate accurate PK parameters.
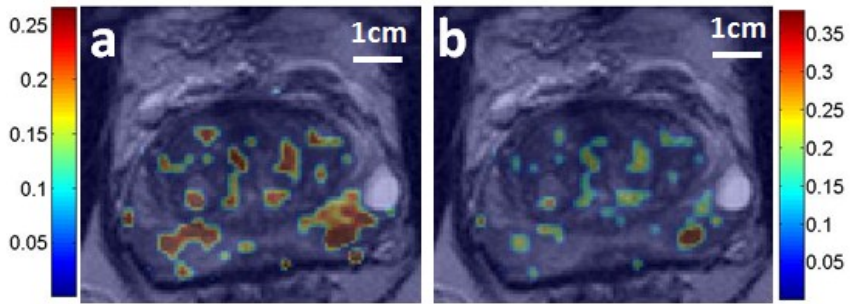

Figure 4. The $K^{\text {trans }}$ map overlaid on a sample DCE-MR image of the prostate calculated using a) the AIF measured from the artery and b) the corrected AIF of the artery.

Figure 2a shows that the correction, results in similar curves for both ACICA results and the concentration measured in an artery. However in many other tumors a large artery to be used in the correction method does not exist and thus some approximations have to be made. Moreover, using contrast uptake in an artery requires finding the time lag between the contrast agent arrival in the artery and its arrival in the tissue of interest. This additional parameter makes the system of equations more complex, difficult to solve and prone to error. Our proposed method has the potential to overcome these shortcomings by calculating the contrast concentration in the tissue of interest using the ACICA algorithm.

Multi-parametric MR imaging can be used to define the prostate tumor location. The performance of our method was also examined using multi-parametric MR images. Figure 3 shows the ADC map and T2-weighted image of the prostate. In both of these images there exists a hypo-intense area in the peripheral zone of the prostate which could correspond to a tumor. Follow up studies confirmed presence of a tumor. The $K^{\text {trans }}$ map of the corrected intravascular concentration, measured using ACICA (figure$3 d$ ), shows high values in the same region confirming the results of Multi-parametric MRI analysis.

Figure 4 shows the $K^{\text {trans }}$ maps that resulted from applying the PK analysis using the corrected and uncorrected AIF curves measured in an artery. The results of corrected curve are very similar to the ones obtained by our method. However the results of applying the artery AIF without correction resulted in high $K^{\text {trans }}$ values in several regions of the prostate and thus had a large number of false positives. These results show that correction is required for PK analysis and that calculating intravascular concentration using ACICA can provide good PK parameters. It also simplifies the analysis by removing the parameter associated with the time lag in bolus arrival.

\section{ACKNOWLEDGEMENTS}

We thank the Natural Sciences and Engineering Research Council of Canada (NSERC) for funding this work.

\section{REFERENCES}

[1] A. Jemal, F. Bray, M. M. Center, J. Ferlay, E. Ward and D. Forman, "Global cancer statistics," CA Cancer Journal for Clinicians, vol. 61, pp. 69-90, 2011.

[2] L. Klotz, "Active surveillance versus radical treatment for favorable-risk localized prostate cancer," Current Treatment Options in Oncology, vol. 7, pp. 355-362, 2006.

[3] M. Heisen, X. Fan, J. Buurman, G. S. Karczmar and B. M. Ter Haar Romeny, "The use of a reference tissue arterial input function with low-temporal-resolution DCE-MRI data," Phys. Med. Biol., vol. 55, pp. 4871-4883, 2010.

[4] D. M. McGrath, D. P. Bradley, J. L. Tessier, T. Lacey, C. J. Taylor and G. J. M. Parker, "Comparison of ModelBased Arterial Input Functions for Dynamic ContrastEnhanced MRI in Tumor Bearing Rats," Magnetic Resonance in Medicine, vol. 61, pp. 1173-1184, 2009.

[5] R. Lawaczeck, G. Jost and H. Pietsch, "Pharmacokinetics of Contrast Media in Humans Model With Circulation, Distribution, and Renal Excretion," Invest. Radiol., vol. 46, pp. 576-585, SEP, 2011.

[6] A. Shukla-Dave, N. Lee, H. Stambuk, Y. Wang, W. Huang, H. T. Thaler, S. G. Patel, J. P. Shah and J. A. Koutcher, "Average arterial input function for quantitative dynamic contrast enhanced magnetic resonance imaging of neck nodal metastases." BMC Med Phys, vol. 9, pp. 4, 2009. [7] H. Mehrabian, C. Chandrana, I. Pang, R. Chopra and A. L. Martel, "Arterial input function calculation in dynamic contrast-enhanced MRI: An in vivo validation study using co-registered contrast-enhanced ultrasound imaging," Eur. Radiol., vol. 22, pp. 1735-1747, 2012.

[8] H. Mehrabian, I. Pang, R. Chopra and A. L. Martel, "An adaptive complex independent component analysis to analyze dynamic contrast enhanced-MRI," in Proc. of Int. Symposium on Biomedical Imaging, 2012, pp. 1052-1055.

[9] H. Mehrabian, R. Chopra and A. Martel, "Calculation of intravascular signal in dynamic contrast enhanced MRI using adaptive complex independent component analysis," Medical Imaging, IEEE Transactions on, doi: 10.1109/TMI.2012.2233747, In Press, 2012.

[10] X. Fan, C. R. Haney, D. Mustafi, C. Yang, M. Zamora, E. J. Markiewicz and G. S. Karczmar, "Use of a reference tissue and blood vessel to measure the arterial input function in DCEMRI," MRM, vol. 64, pp. 1821-1826, 2010.

[11] P. Comon, "Independent component analysis, A new concept?" Signal Process, vol. 36, pp. 287-314, 1994.

[12] M. Novey and T. Adali, "Complex ICA by negentropy maximization," IEEE Trans. Neural Networks, vol. 19, pp. 596-609, 2008.

[13] P. S. Tofts and A. G. Kermode, "Measurement of the Blood-Brain-Barrier Permeability and Leakage Space using Dynamic Mr Imaging .1. Fundamental-Concepts," Magnetic Resonance in Medicine, vol. 17, pp. 357-367, 1991. 